\title{
CORRECTION
}

View Article Online

View Journal I View Issue

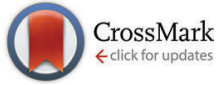

Cite this: New J. Chem., 2016, 40,1882

DOI: $10.1039 / c 6 n j 90005 d$

www.rsc.org/njc

\section{Correction: Light-penetration and light-scattering effects in dye-sensitised solar cells}

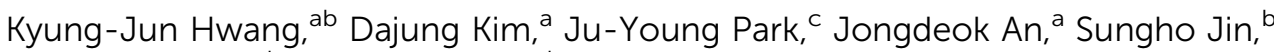 \\ Sang Ook Kang, ${ }^{d}$ Dae Won Cho*ad and Chan Im*ae
}

Correction for 'Light-penetration and light-scattering effects in dye-sensitised solar cells' by Kyung-Jun Hwang et al., New J. Chem., 2014, 38, 6161-6167.

The authors would like to note that a significant proportion of the data reported in the above paper was collected at Konkuk University-Fraunhofer ISE Next Generation Solar Cell Research Center (KFnSC), Konkuk University, without the appropriate acknowledgement of this institution and the researchers therein. To correct this, the authors would like to make the following changes:

Dajung Kim, Jongdeok An and Chan Im contributed to this work, but their contribution was not acknowledged in the original report. These three researchers have been added to the author list, as indicated above.

KFnSC has been added as an additional affiliation for Kyung-Jun Hwang and Dae Won Cho, as indicated above.

The authors would like to amend the acknowledgements to:

This research was supported by Seoul R\&BD Program (WR090671). This research was also supported by the Basic Science Research Program through the National Research Foundation of Korea (NRF) funded by the Ministry of Education (NRF-2013R1A6A3A03021144, K.-J. Hwang and NRF-2014R1A1A-2004199, D. W. Cho).

The authors would like to note that Kyung-Jun Hwang and Dajung Kim contributed equally to the original work.

The Royal Society of Chemistry apologises for these errors and any consequent inconvenience to authors and readers.

\footnotetext{
${ }^{a}$ Konkuk University-Fraunhofer ISE Next Generation Solar Cell Research Center(KFnSC), Konkuk University, Seoul 143-701, Korea. E-mail: trapezium_9@naver.com, anhijuk@konkuk.ac.kr

${ }^{b}$ Mechanical and Aerospace Engineering, University of California at San Diego, La Jolla, CA 92093, USA. E-mail: kyhwang@ucsd.edu, jin@ucsd.edu

${ }^{c}$ Department of Electrical Engineering, Chonnam National University, Gwangju 500-757, Korea. E-mail: gom8812@hanmail.net

${ }^{d}$ Department of Advanced Materials Chemistry, Korea University (Sejong Campus), Sejong 339-70o, Korea. E-mail: sangok@korea.ac.kr, dwcho@korea.ac.kr

${ }^{e}$ Department of Chemistry, Konkuk University, Seoul 143-701, Korea. E-mail: chanim@konkuk.ac.kr
} 